\section{Death by Nondiagnosis: Why Emergent CT Angiography Should Not Be Done for Patients with Subarachnoid Hemorrhage}

We read with great interest the recent editorial in this journal by Kallmes et $\mathrm{al}^{1}$ about CT angiography (CTA) in patients with subarachnoid hemorrhage ( $\mathrm{SAH}$ ). As can be expected in an editorial, the content is provocative because the authors state that conventional angiography is the one and only diagnostic in the work-up of patients with the suspicion of an aneurysmal SAH and that CT angiography (as a modern "toy") is inaccurate and potentially deadly for the patients. The authors state that conventional angiography has near-perfect accuracy and a very low mortality rate $(0.06 \%)$. CTA is falsenegative in $10 \%$ of cases, and "back of the envelope calculation" resulted in "an additional 2.5 deaths per 100 patients from rehemorrhage of undiagnosed ruptured aneurysms" with CTA. Also, finance is an issue because the authors wrote, "For our money, we'll take conventional angiography any day over CTA."1

In the December 2007 issue of Neuroradiology, ${ }^{2}$ we reported about our experience with both 16- and 64-detector-row CTA as the first and intended only diagnostic and treatment decision-making study for intracranial aneurysms in 224 patients with acute SAH in the clinical setting of a primary and secondary neurosurgical referral center. Patients with an SAH are considered a neurosurgical case, with a multidisciplinary team (neurosurgeon, neurologist, neuroradiologist, and neuroanesthesiologist) taking care of proper state-of the-art treatment. Every patient is checked with a plain CT scan immediately after admission and also when a previous CT scan from a referring center is available, to rule out additional problems that might need acute treatment (ie, subacute hydrocephalus, rebleed, intracerebral hematoma). In the next run, CTA is performed. CTA seemed to have $96 \%$ accuracy in the detection of symptomatic aneurysms. Additional conventional angiography (which has been the method of first choice in the past in our department as well) seemed to have marginal complementary value, except for patients with nonperimesencephalic SAH or negative or inconclusive CTA results. The risk of rebleeding after a negative initial CTA result was 7\%. In all patients with a rebleeding conventional angiography, results were also false-negative. ${ }^{2}$

Other studies with use of conventional angiography as the first diagnostic tool report similar findings. ${ }^{3,4}$ Multidetector CTA has an excellent spatial resolution with isotropic voxel resolutions $(0.5 \mathrm{~mm}$ in $\mathrm{x}$ - and $\mathrm{y}$-axis and $0.6 \mathrm{~mm}$ in $\mathrm{z}$-axis for 16-section CTA and $0.4 \mathrm{~mm}$ in $x-, y-$, and $z$-axis for 64-section CTA). Therefore, false-negative findings are not a reflection of lack of spatial resolution if multidetector CT systems of higher-order are used rather than those of 16-row (ie, enables isotropic resolutions). The references of Dr. Kallmes are unpublished data and refer to presentations in proceedings reports of data of nonisotropic resolution. On the basis of these results, no conclusions can be drawn whatsoever. We are convinced that Kallmes et al will be able to recall from their own practice similar examples of patients with fatal rebleeds after high-tech digital subtraction angiography (DSA). Even to date, one has to accept that some cerebral aneurysms are occult on initial, and even on follow-up, angiography. It is surprising that the authors seem to completely ignore the risks that are related to endovascular angiography. DSA carries a risk for neurologic complications of $2.6 \%$, with permanent deficit in $0.14 \%$ of cases. ${ }^{5}$ This should be taken into account when deciding for routine DSA in the work-up of patients suspected of having an aneurysmal SAH.

On the basis of our data, we feel confident replacing conventional angiography with CTA as the first diagnostic technique in the evaluation of patients with an SAH. CTA seemed to be a reliable, fast, easy-to-do, and minimally invasive imaging technique, helping to avoid a number of the well-known, procedure-related risks for invasive conventional angiography. The implementation of routine CTA in the investigation of cerebral aneurysms has offset some of the workload while improving workflow. Last, but not least, the costs of CTA are considerably lower compared with conventional angiography.

In our opinion, the time-consuming, more risky, and more expensive technique of conventional angiography should be reserved for a selected number of cases with negative or inconclusive findings on CTA in patients with a SAH.

\section{References}

1. Kallmes DF, Layton K, Marx WF, et al. Death by nondiagnosis: why emergent CT angiography should not be done for patients with subarachnoid hemorrhage. AJNR Am J Neuroradiol 2007;28:1837-38

2. Westerlaan HE, Gravendeel J, Fiore D, et al. Multislice CT angiography in the selection of patients with ruptured intracranial aneurysms suitable for clipping or coiling. Neuroradiology 2007;49:997-1007

3. Urbach H, Zentner J, Solymosi L. The need for repeat angiography in subarachnoid haemorrhage. Neuroradiology 1998;40:6-10

4. Bradac GB, Bergui M, Ferrio MF, et al. False-negative angiograms in subarachnoid haemorrhage due to intracranial aneurysms. Neuroradiology 1997;39:772-76

5. Kaufmann TJ, Huston J 3rd, Mandrekar JN, et al. Complications of diagnostic cerebral angiography: evaluation of 19,826 consecutive patients. Radiology 2007;243:812-19

H.E. Westerlaan

S. Eshghi

M. Oudkerk

Department of Radiology

R.J.M. Groen

J.D.M. Metzemaekers

J.M.C. van Dijk

J.J.A. Mooij

Department of Neurosurgery University Medical Center Groningen Groningen, The Netherlands

DOI 10.3174/ajnr.A1011 